UT-938

\title{
Nonanomalous R-symmetry in supersymmetric unified theories of quarks and leptons
}

\author{
Kiichi Kurosawa, ${ }^{1}$ Nobuhito Maru $^{1}$ and T. Yanagida ${ }^{1,2}$ \\ 1 Department of Physics, University of Tokyo, Tokyo 113-0033, Japan \\ ${ }^{2}$ Research Center for the Early Universe, University of Tokyo, Tokyo 113-0033, Japan
}

\begin{abstract}
A discrete R-symmetry often appears as an exact gauge symmetry in the low energy effective theory of superstring theories. We search for such discrete Rsymmetries from a phenomenological point of view and find that $Z_{9 R}$ and $Z_{18 R}$ are candidates of the nonanomalous R-symmetry in the case of the minimal supersymmetric standard model. We also find $Z_{4 R}$ and $Z_{20 R}$ in the case that quarks and leptons are embedded in the SU(5) GUT multiplets. Interesting is that in the latter case all the solutions predict some extra matter multiplets and we find that the simplest choice of the extra matters is to take a pair of $\mathbf{5}$ and $\mathbf{5}^{*}$ of $\mathrm{SU}(5)_{\mathrm{GUT}}$ whose mass is of order the SUSY breaking scale $\sim 1 \mathrm{TeV}$. We emphasize that the presence of such extra matters is testable in future hadron collider experiments.
\end{abstract}




\section{Introduction}

Higher dimensional supergravity theories such as superstring theories always contain R-symmetry, which is naturally broken down to its discrete subgroup $Z_{N R}$ by the compactification of the extra space [1, 2]. This discrete R-symmetry should be nonanomalous since it is a gauge symmetry. Thus, the presence of a nonanomalous discrete $Z_{N R}$ is one of important low-energy ingredients of higher dimensional supergravity. On the other hand, the discrete R-symmetry is a crucial symmetry that suppresses the dangerous dimension-four operators [3, 4] causing too rapid proton decays and hence it guarantees the matter stability in the supersymmetric standard model (SUSY SM). This discrete R-symmetry is also very important to understand the light Higgs doublet in the standard model. That is, the discrete R-symmetry may forbid the SUSY-invariant mass term ( $\mu$-term) of the Higgs chiral multiplets. Furthermore, when the R-symmetry breaking is linked to the SUSY breaking the Higgs chiral multiplets acquire a SUSY-invariant mass of order the gravitino mass $m_{3 / 2} \sim 1 \mathrm{TeV}$ [0].円

Our question is whether such a nonanomalous discrete R-symmetry exists or not in a phenomenological point of view.2 We show in this paper that it indeed exists in the minimal SUSY standard model (MSSM) and that the nonanomalous discrete R-symmetry is restricted to either $Z_{9 R}$ or $Z_{18 R}$. [ However, we point out that the discrete R-symmetry is always anomalous if we take the R-charge assignment consistent with the grand unification model (GUT) of quarks and leptons. We also find that the anomalies are cancelled out by a pair of extra matter multiplets 5 and $\mathbf{5}^{*}$ of SU(5) $)_{\text {GUT }}$ at the SUSY-breaking scale $\sim 1 \mathrm{TeV}$, and that the allowed R-symmetries are $Z_{4 R}$ or $Z_{20 R}$. We explicitly construct a unification model based on a semi-simple gauge group $\mathrm{SU}(5)_{\mathrm{GUT}} \times \mathrm{U}(3)_{\mathrm{H}}[8,9,10$.

\footnotetext{
${ }^{1}$ Throughout this paper we assume that the SUSY breaking is mediated to the MSSM sector by nonrenormalizable interactions suppressed by the Planck scale.

${ }^{2}$ We mean by "nonanomalous" that anomalies are cancelled out among only fermions. In this paper we do not pursue a possibility of anomaly cancellations by the Green-Schwartz mechanism [6].

${ }^{3}$ A related topic is discussed by Ibanez and Ross in Refs. [7]. Our point is that we can say more about the discrete R-symmetry by focusing on the above relation between the SUSY breaking and the $\mu$-term of the Higgs multiplets.
} 


\begin{tabular}{|c||c|c|c|c|c|c|c|c||c|}
\hline & $Q$ & $\bar{U}$ & $\bar{D}$ & $L$ & $\bar{E}$ & $\bar{N}$ & $H$ & $\bar{H}$ & $\theta$ \\
\hline $\mathrm{SU}(3)_{C}$ & $\mathbf{3}$ & $\mathbf{3}^{*}$ & $\mathbf{3}^{*}$ & $\mathbf{1}$ & $\mathbf{1}$ & $\mathbf{1}$ & $\mathbf{1}$ & $\mathbf{1}$ & \\
\hline $\mathrm{SU}(2)_{L}$ & $\mathbf{2}$ & $\mathbf{1}$ & $\mathbf{1}$ & $\mathbf{2}$ & $\mathbf{1}$ & $\mathbf{1}$ & $\mathbf{2}$ & $\mathbf{2}$ & \\
\hline $\mathrm{U}(1)_{Y}$ & $1 / 6$ & $-2 / 3$ & $1 / 3$ & $-1 / 2$ & 1 & 0 & $1 / 2$ & $-1 / 2$ & \\
\hline$Z_{N R}$ & $q$ & $\bar{u}$ & $\bar{d}$ & $l$ & $\bar{e}$ & $\bar{n}$ & $h$ & $\bar{h}$ & $\alpha$ \\
\hline
\end{tabular}

Table 1: The matter content of the MSSM. $\theta$ is the Grassmann coordinate. All $Z_{N R}$ charges are taken to be integer. If one chooses the $Z_{N R}$ charge of the Grassmann coordinate $\theta$ to be unity as done in most of literatures, the $Z_{N R}$ charges of the chiral multiplets should be taken fractional. We consider only a generation independent $Z_{N R}$ symmetry in this paper, so generation indices are omitted.

\section{Discrete R-symmetry in MSSM}

We first discuss conditions that an anomaly-free discrete R-symmetry $Z_{N R}$ should satisfy in the MSSM (including right-handed neutrinos). Then we show that such an Rsymmetry does exist and that it is restricted to either $Z_{9 R}$ or $Z_{18 R}$. We also discuss their phenomenological aspects.

The MSSM matter content and the $Z_{N R}$ charges are listed in the Table 1. It is assumed through this paper that tiny neutrino masses are generated by the see-saw mechanism [11]. Then the superpotential in the MSSM is

$$
W=Q \bar{U} H+Q \bar{D} H+L \bar{E} \bar{H}+L \bar{N} \bar{H}+\frac{1}{2} M_{m} \bar{N} \bar{N}+\mu H \bar{H},
$$

where $M_{m}$ is a Majorana mass for the right-handed neutrinos and $\mu$ is a SUSY-invariant mass for the Higgs doublets. Since the superpotential has the $Z_{N R}$ charge $2 \alpha$ it imposes the following conditions for the $Z_{N R}$ charges,

$$
\begin{aligned}
q+\bar{u}+h & =2 \alpha \bmod N, \\
q+\bar{d}+\bar{h} & =2 \alpha \bmod N, \\
l+\bar{e}+\bar{h} & =2 \alpha \bmod N, \\
l+\bar{n}+h & =2 \alpha \bmod N, \\
2 \bar{n} & =2 \alpha \bmod N,
\end{aligned}
$$

\footnotetext{
${ }^{4}$ Here we have assumed that the $Z_{N R}$ charge of the Majorana mass $M_{m}$ is zero, that is, the generation of the Majorana mass scale is independent of the SUSY breaking.
} 
As for the last term in the superpotential, $\mu$ must be of the order of the weak scale. One of the most attractive mechanisms to generate the $\mu$-term is the Giudice-Masiero mechanism [5]. That is, the Kähler potential,

$$
K=\kappa H \bar{H}
$$

induces the $\mu$-term of the weak scale,

$$
W=\kappa\langle W\rangle H \bar{H},
$$

where $\kappa$ is $\mathcal{O}(1)$ constant and $\langle W\rangle=m_{3 / 2} \sim 1 \mathrm{TeV}$. Hereafter, we take the Planck scale $M_{P}=2.4 \times 10^{18} \mathrm{GeV}$ to be unity. In order to obtain the $\mu$-term through the Giudice-Masiero mechanism the following conditions have to be imposed:

$$
\begin{aligned}
h+\bar{h} & =0 \bmod N, \\
h+\bar{h} & \neq 2 \alpha \bmod N .
\end{aligned}
$$

Eq.(10) is necessary to prevent the Higgs doublets from having a Planck scale mass. It can be rewritten as

$$
2 \alpha \neq 0 \bmod N
$$

from Eq. (9). This condition is also desirable in viewpoint of the cosmological constant problem since $\langle W\rangle=0$ as long as the $Z_{N R}$ symmetry is unbroken. Otherwise, we have $\langle W\rangle=\mathcal{O}(1)$ leading to a negative cosmological constant of the order of the Planck scale [10]. Furthermore, this condition excludes the $N=2$ case including the R-parity, so that $N \geq 3$.

The anomaly cancellation conditions we should consider [7] are

$$
\begin{aligned}
& \mathcal{A}_{3}=\frac{3}{2}\{2(q-\alpha)+(\bar{u}-\alpha)+(\bar{d}-\alpha)\}+3 \alpha=\frac{N}{2} k, \\
& \mathcal{A}_{2}=\frac{3}{2}\{3(q-\alpha)+(l-\alpha)\}+\frac{1}{2}\{(h-\alpha)+(\bar{h}-\alpha)\}+2 \alpha=\frac{N}{2} k^{\prime},
\end{aligned}
$$

\footnotetext{
${ }^{5}$ The mixed anomaly $Z_{N R}\left[\mathrm{U}(1)_{Y}\right]^{2}$ does not give any useful condition because the $\mathrm{U}(1)_{Y}$ charge is not quantized [7, 12].
} 
where $\mathcal{A}_{3}$ and $\mathcal{A}_{2}$ are anomaly coefficients for $Z_{N R}\left[\mathrm{SU}(3)_{C}\right]^{2}$ and $Z_{N R}\left[\mathrm{SU}(2)_{L}\right]^{2}$, respectively. $k$ and $k^{\prime}$ are integers. These conditions are simplified to

$$
\begin{aligned}
2 q+\bar{u}+\bar{d} & =2 \alpha+\frac{N}{3} k, \\
3(3 q+l)+h+\bar{h} & =10 \alpha+N k^{\prime} .
\end{aligned}
$$

Using Eqs.(2), (3) and (9) we obtain

$$
2 q+\bar{u}+\bar{d}=4 \alpha \quad \bmod N .
$$

Then the anomaly cancellation condition for $Z_{N R}\left[\mathrm{SU}(3)_{C}\right]^{2}$ in Eq.(12) becomes

$$
N=\frac{6 \alpha}{k} .
$$

Together with Eqs.(5)-(9), on the other hand, the anomaly cancellation condition for $Z_{N R}\left[\mathrm{SU}(2)_{L}\right]^{2}$ becomes

$$
9 q-3 h-7 \alpha=N k^{\prime}+\eta \frac{N}{2} k^{\prime \prime},
$$

where $k^{\prime \prime}$ is an integer and $\eta=0$ or 1 for $N$ being odd or even. Note that the integer $k$ should not be a multiple of three from Eqs.(11) and (17). Then $N$ must be a multiple of three. It, however, imposes that the integer $\alpha$ has to be a multiple of three from Eq.(18). At last it is found that $N=9 M$, where $M$ is an integer.

Finally, we discuss the other anomaly cancellation conditions. The cancellation condition of the mixed gravitational anomaly [7] is given as follows:

$$
\begin{aligned}
\mathcal{A}_{\text {gravity }}= & 18(q-\alpha)+9(\bar{u}-\alpha)+9(\bar{d}-\alpha)+6(l-\alpha)+3(\bar{e}-\alpha)+3(\bar{n}-\alpha) \\
& +2(h-\alpha)+2(\bar{h}-\alpha)+(8+3+1-21) \alpha \\
= & -13 \alpha=m N+\eta n \frac{N}{2},
\end{aligned}
$$

where $m, n$ are integers. For $N=$ odd case,

$$
\mathcal{A}_{\text {gravity }}=5 \alpha \bmod \frac{6 \alpha}{k} \text {. }
$$

For $N=$ even case,

$$
\mathcal{A}_{\text {gravity }}=2 \alpha \bmod \frac{3 \alpha}{k} \text {. }
$$


One can see that the MSSM matter content does not cancel the mixed gravitational anomaly. However, it is easy to cancel this anomaly by introducing five (two) singlets with vanishing $\mathrm{R}$-charges for $N=$ odd (even), respectively. These singlets have masses of the order of the gravitino mass through the Giudice-Masiero mechanism like the Higgs doublets. The cubic anomaly $Z_{N}^{3}$ quite depends on the charges of heavy fields. Thus, it is not so useful to constrain the low energy physics as long as we have no information about the massive sector [7, 12]. Indeed, we have checked that the cubic anomalies are not cancelled by only the MSSM matter content (and the above mentioned singlets) but cancelled by adding some extra heavy fields with fractional charges.

To summarize, we have above shown that the discrete $Z_{N R}$ symmetry should satisfy the following eight conditions: four from the Yukawa couplngs (Eqs.(2)-(5)), one from the Majorana mass for the right-handed neutrinos (Eq.([6]), one from the Giudice-Masiero mechanism (Eq.(9)), and two from the anomaly cancellation conditions (Eqs.(12) and (13)). Moreover, note that there is one constraint (Eq.(11)) from the Giudice-Masiero mechanism.

Before solving the above eight conditions, we show that the number of independent $Z_{N R}$ charge assignments is finite. Since there are ten free parameters (nine charges given in Table 1 and one parameter $N$ ), it seems that there are two free parameter and that the number of allowed charge assignments is infinite. However, these assignments are not independent of each other. First, the following shift of a $Z_{N R}$ charge assignment by the integer hypercharge (the hypercharge multiplied by six) gives a physically equivalent charge assignment because of the presence of the $\mathrm{U}(1)_{Y}$ gauge symmetry [7]:

$$
\begin{aligned}
\mathcal{Q} \rightarrow & \mathcal{Q}+(1,-4,2,-3,6,0,3,-3,0) . \\
& (\mathcal{Q} \equiv(q, \bar{u}, \bar{d}, l, \bar{e}, \bar{n}, h, \bar{h}, \alpha))
\end{aligned}
$$

Second, there is a freedom of total normalization of the $Z_{N R}$ charges. Consequently, the number of independent charge assignments is finite and indeed only twelve as will be shown below.

Now we present the explicit $Z_{N R}$ charge assignments. As in Ref.[0], it is convenient

\footnotetext{
${ }^{6}$ There is another possibility to cancel the mixed gravitational anomaly. This is done by introducing only one singlet with R-charge $2 \alpha$ for $N=$ odd and even cases. In this case, this singlet remains massless.
} 


\begin{tabular}{|l|rrrrrrrrr|}
\hline & $q$ & $\bar{u}$ & $\bar{d}$ & $l$ & $\bar{e}$ & $\bar{n}$ & $h$ & $\bar{h}$ & $\alpha$ \\
\hline$P$ & 1 & 1 & 1 & 1 & 1 & 1 & 0 & 0 & 1 \\
$R$ & 0 & -1 & 1 & 0 & 1 & -1 & 1 & -1 & 0 \\
$L$ & 0 & 0 & 0 & -1 & 1 & 1 & 0 & 0 & 0 \\
\hline
\end{tabular}

Table 2: The charges of the three generators. $P, R$ and $L$ correspond to the $\mathcal{R}$-symmetry (the generalized R-parity), the right-handed isospin and the lepton number, respectively.

to represent the $Z_{N R}$ charge assignments using a linear combination of some independent generators such as the lepton number. First we use five of eight conditions, that is, four from the Yukawa couplings and one from the Giudice-Masiero mechanism. In addition, we set the charge $q$ equal to $\alpha$ using the shift given in Eq.(22). Since three charges remain undetermined, we choose the $\mathcal{R}$-symmetry, the right-handed isospin and the lepton number given in Table 2 as three independent generators. Then the $Z_{N R}$ charge assignments are represented by a linear combination of these three generators.

$$
Z_{N R}: P^{\alpha} R^{\beta} L^{\gamma} \quad(\alpha, \beta, \gamma=0,1, \cdots, N-1)
$$

This expression means that the $Z_{N R}$ charges are assigned as $\mathcal{Q}=\alpha P+\beta R+\gamma L$. The three integers $\alpha, \beta$ and $\gamma$ are determined by the remaining three conditions and one constraint as follows:

$$
\begin{aligned}
6 \alpha & =0 \bmod N, \\
-2 \beta+2 \gamma & =0 \bmod N, \\
2 \alpha-3 \gamma & =0 \bmod N, \\
2 \alpha & \neq 0 \bmod N .
\end{aligned}
$$

Solving these four equations and removing ambiguity of total normalization of the charges, we find that $N=9$ or 18 and obtain the following twelve charge assignments:

$$
\begin{aligned}
Z_{9 R} & : P^{3}(R L)^{3 m+2}, \\
Z_{18 R} & : \quad P^{3}(R L)^{6 m+2}, \quad P^{3}(R L)^{6 m+2} R^{9}, \quad P^{6}(R L)^{6 m+4} R^{9},
\end{aligned}
$$

where $m=0,1,2$. Implications of these charge assignments are understood as follows. Recall the equivalence of the shift by the hypercharge $Y$. That is, the generator $R L$ is 
equivalent to the baryon number $B=R L Y^{-2}$, and the generator $R^{9}$ is the matter parity $\left(R Y^{-6}\right)^{9}$. Therefore, the above $Z_{N R}$ charge assignments are understood as the combination of the $\mathcal{R}$-symmetry and the baryon number (plus the matter parity for $Z_{18 R}$ ). $L$ And the patterns of these combinations are determined by the anomaly cancellation conditions.

One can easily check that these discrete R-symmetries forbid all of the dimension four and five operators which violate the baryon number or the lepton number except for the operator $L L H H$. In the case of $Z_{9 R}$, for example, an allowed operator has to satisfy the following condition:

$$
3 Q_{P}+(3 m+2) Q_{B}=6 \bmod N(=9)
$$

where $Q_{P}$ is the $\mathcal{R}$-charge of the operator $\left(Q_{P}=1\right.$ for the Grassmann coordinate $\left.\theta\right)$ and $Q_{B}$ the baryon number. Obviously, the unwanted dimension four and five operators cannot satisfy the above condition.

When SUSY is broken, the $Z_{9 R}$ and $Z_{18 R}$ symmetries are spontaneously broken by the condensation of the superpotential $\langle W\rangle \neq 0$. The superpotential has a $Z_{N R}$ charge $2 \alpha=6\left(Z_{9 R}\right.$ and the first two of $Z_{18 R}$ 's) or $2 \alpha=12$ (the last one of $Z_{18 R}$ 's). Therefore, the $Z_{9 R}$ and $Z_{18 R}$ symmetries are broken down as follows:

$$
\begin{aligned}
Z_{9 R} \rightarrow Z_{3} & :(R L)^{2} \\
Z_{18 R} \rightarrow Z_{6 R} & : \quad P^{3}(R L)^{2}, P^{3}(R L)^{2} R^{3} \\
Z_{6} & :(R L)^{4} R^{3}
\end{aligned}
$$

Although the order parameter of the SUSY breaking is $\langle W\rangle \sim 10^{-15}$, it might bring about significant cosmological signatures through the effective dimension four operators. In the cases of $\mathrm{Z}_{9 R}$ and the last of $\mathrm{Z}_{18 R}$ 's there remains no discrete R-symmetry. Then the lepton number violating dimension four operators $(Q \bar{D} L, L L \bar{E})$ appear with a coupling $\lambda \sim\langle W\rangle \sim 10^{-15}$, so that the lightest SUSY particle (LSP) is unstable. If the LSP is

\footnotetext{
7 The lepton number and the Peccei-Quinn symmetry are violated by the Majorana mass term and the Giudice-Masiero mechanism, respectively. This is why the above charge assignments are given by combinations of the $R$-symmetry and the baryon number.

8 The baryon number violating one $(\bar{U} \bar{D} \bar{D})$ is still forbidden, so that there are no proton decays through the dimension four operators.
} 
the photino as in most gravity-mediated SUSY breaking models, its lifetime [13] is

$$
\tau_{L S P} \sim\left(\frac{\alpha \lambda^{2}}{128 \pi^{2}} \frac{m_{L S P}^{5}}{m_{\tilde{f}}^{4}}\right)^{-1} \sim\left(\frac{\lambda}{10^{-15}}\right)^{-2} \times 10^{9} \mathrm{~s},
$$

where $m_{\tilde{f}}$ is the related squark or slepton mass and in the last equality we have assumed that $m_{L S P} \sim m_{\tilde{f}} \sim 100 \mathrm{GeV}$. The LSP with a life time of order $10^{9} s$ is excluded because it causes a significant distortion of cosmic microwave background or spoils the success of the big bang nucleosynthesis [14]. Even if the coupling $\lambda$ are further suppressed and the lifetime of the LSP is longer than the age of the universe, the absence of a high energy neutrino background requires that $\tau_{L S P}>10^{17} \mathrm{yr}$ [15, which imposes an unnatural tuning on the coupling $\lambda<10^{-23}$.

Consequently, the first two charge assignments of $Z_{18 R}$ in Eq.(30) are phenomenologically viable. In these cases a discrete $R$ symmetry $Z_{6 R}$ remains unbroken and then the unwanted dimension four operators are absent even after the SUSY breaking.

\section{GUT and discrete R-symmetry}

In this section, we extend the previous analysis to the case that the $Z_{N R}$ charge assignment is consistent with the grand unification model (GUT) of quarks and leptons. That is, we assign the same $Z_{N R}$ charges to the quarks and leptons in the same multiplet of $\mathrm{SU}(5)_{\mathrm{GUT}}$ as follows:

$$
\begin{aligned}
t & \equiv q=\bar{u}=\bar{e} \\
\bar{f} & \equiv \bar{d}=l .
\end{aligned}
$$

Then the conditions Eqs.(2)-(6) are rewritten as

$$
\begin{aligned}
2 t+h & =2 \alpha \bmod N, \\
t+\bar{f}+\bar{h} & =2 \alpha \bmod N \\
\bar{f}+\bar{n}+h & =2 \alpha \bmod N \\
2 \bar{n} & =2 \alpha \bmod N .
\end{aligned}
$$

In addition, Eqs.(9) and (10) have to be imposed for the Giudice-Masiero mechanism to work. In the $\mathrm{SU}(5)_{\text {GUT }}$ respected case the anomaly cancellation conditions Eqs. (12) 
and (13) are obtained as

$$
\begin{aligned}
& \mathcal{A}_{3}=3 \alpha=\frac{N}{2} k \\
& \mathcal{A}_{2}=\alpha=\frac{N}{2} k^{\prime}
\end{aligned}
$$

from Eqs.(9), (38) and (39).

Notice that Eq.(43) contradicts Eq.(11). This contradiction implies that there are some extra matter multiplets contributing to the anomalies than the MSSM particles. Since these extra matter multiplets must form complete multiplets of the $\mathrm{SU}(5)_{\mathrm{GUT}}$ in order not to spoil the gauge coupling unification, they equally contribute to the anomaly coefficients for $\mathrm{SU}(3)_{C}$ and $\mathrm{SU}(2)_{L}$. Therefore, the difference between these two anomaly coefficients $\mathcal{A}_{3}$ and $\mathcal{A}_{2}$ is independent of the choice of the extra matter multiplets, that is, the following condition has to be satisfied

$$
\mathcal{A}_{3}-\mathcal{A}_{2}=2 \alpha=\frac{N}{2} k^{\prime \prime}
$$

Since the integer $k^{\prime \prime}$ must be odd from Eq.(11), it is found that $N=4 M$.

Obviously, the above extra matter multiplets are massless when the discrete Rsymmetry $Z_{N R}$ is exact. The Giudice-Masiero mechanism seems the most natural way to induce their masses larger than the direct experimental bounds. Therefore, the extra matter multiplets should satisfy the condition

$$
x+\bar{x}=0 \bmod N,
$$

where $x$ and $\bar{x}$ are the $Z_{N R}$ charge of the extra matter multiplets $\Psi$ and $\bar{\Psi}$, respectively. For example, if we take one pair of 5 and $5^{*}$ in the $\mathrm{SU}(5)_{\text {GUT }}$ as the extra matter multiplets, they contribute to the anomaly coefficients $\mathcal{A}_{3}$ and $\mathcal{A}_{2}$ by $-\alpha$. Thus, it is found that this simplest choice is enough to make the $Z_{N R}$ symmetry nonanomalous.

By the same arguments as in the MSSM, we can find that only $Z_{4 R}$ and $Z_{20 R}$ are independent and that the corresponding charge assignments are as follows:

$$
\begin{aligned}
Z_{4 R} & : P V^{2 m} \quad(m=0,1) \\
Z_{20 R} & : \quad P^{5} V^{2 m} \quad(m=1,2,3,4,6,7,8,9)
\end{aligned}
$$




\begin{tabular}{|l|rrrrrr|}
\hline & $t$ & $\bar{f}$ & $\bar{n}$ & $h$ & $\bar{h}$ & $\alpha$ \\
\hline$P$ & 1 & 1 & 1 & 0 & 0 & 1 \\
$V$ & 1 & -3 & 5 & -2 & 2 & 0 \\
\hline
\end{tabular}

Table 3: The charges of the two generators. $P$ is the same as in Table 2. The generator $V$ corresponds to a $\mathrm{U}(1)$ subgroup of $\mathrm{SO}(10)\left(\supset \mathrm{SU}(5)_{\mathrm{GUT}} \times \mathrm{U}(1)_{V}\right)$.

\begin{tabular}{|c|ccccc|ccccc|c|}
\hline & $T_{r s}$ & $\bar{F}^{r}$ & $\bar{N}$ & $H_{r}$ & $\bar{H}^{r}$ & $Q_{\alpha}^{r}$ & $\bar{Q}_{r}^{\alpha}$ & $Q_{\alpha}^{6}$ & $\bar{Q}_{6}^{\alpha}$ & $X_{\beta}^{\alpha}$ & $\theta$ \\
\hline $\mathrm{SU}(5)_{\mathrm{GUT}}$ & $\mathbf{1 0}$ & $\mathbf{5}^{*}$ & $\mathbf{1}$ & $\mathbf{5}$ & $\mathbf{5}^{*}$ & $\mathbf{5}^{*}$ & $\mathbf{5}$ & $\mathbf{1}$ & $\mathbf{1}$ & $\mathbf{1}$ & \\
\hline $\mathrm{SU}(3)_{H}$ & $\mathbf{1}$ & $\mathbf{1}$ & $\mathbf{1}$ & $\mathbf{1}$ & $\mathbf{1}$ & $\mathbf{3}$ & $\mathbf{3}^{*}$ & $\mathbf{3}$ & $\mathbf{3}^{*}$ & $\mathbf{8}$ & \\
\hline $\mathrm{U}(1)_{H}$ & 0 & 0 & 0 & 0 & 0 & 1 & -1 & 1 & -1 & 0 & \\
\hline$Z_{4 R}$ & 1 & 1 & 1 & 0 & 0 & 0 & 0 & 2 & 2 & 2 & 1 \\
\hline
\end{tabular}

Table 4: The matter content in the $\mathrm{SU}(5)_{\mathrm{GUT}} \times \mathrm{U}(3)_{H}$ model.

where the generators $P$ and $V$ are defined in Table 3. $V^{2 m}$ can be understood as a remaining discrete subgroup of $\mathrm{U}(1)_{V}$ after generation of right-handed Majorana neutrino masses: $\mathrm{U}(1)_{V} \rightarrow Z_{10 V}$. Therefore, if we gauge this $\mathrm{U}(1)_{V}$ as in the $\mathrm{SO}(10)_{\mathrm{GUT}}$, the above ten discrete R-symmetries are equivalent to each other, and the nonanomalous discrete R-symmetry is uniquely determined as $\mathrm{Z}_{4 R}: P$.

It can be easily checked that these discrete R-symmetries forbid all the unwanted dimension four and five operators. This is because an allowed operator has to satisfy the condition $Q_{P}-2 m\left(Q_{B}-Q_{L}\right)=2(\bmod 4)$, where $Q_{L}$ is the lepton number. (Note that the generator $V$ is equivalent to $\left(B^{-1} L\right)^{5}$ up to the $\mathrm{U}(1)_{Y}$ gauge symmetry.) After the SUSY breaking both $Z_{4 R}$ and $Z_{20 R}$ is broken down to $Z_{2 R}$ (the R-parity), so that the unwanted dimension four operators are forbidden completely.

We have embedded quarks and leptons in the GUT multiplets. As for the Higgs sector, however, a naive extension to the GUT multiplets brings about massless colored Higgs multiplets. The only model free from this problem is the "R-invariant natural unification" model with a semisimple gauge group $\mathrm{SU}(5)_{\mathrm{GUT}} \times \mathrm{U}(3)_{H}$ [8, 9, 10]. The matter content in this model is given in Table 4 , where we take $Z_{4 R}$ as the discrete R-symmetry for simplicity. The superpotential in this model is 


$$
W=Q_{\alpha}^{r} \bar{Q}_{r}^{\beta} X_{\beta}^{\alpha}+Q_{\alpha}^{6} \bar{Q}_{6}^{\beta} X_{\beta}^{\alpha}+Q_{\alpha}^{r} \bar{Q}_{6}^{\alpha} H_{r}+\bar{Q}_{r}^{\alpha} Q_{\alpha}^{6} \bar{H}^{r} .
$$

As shown in Ref. [10] only $Q_{\alpha}^{r}$ and $\bar{Q}_{r}^{\alpha}$ acquire the vacuum expectation value $v$,

$$
\left\langle Q_{\alpha}^{r}\right\rangle=v \delta_{\alpha}^{r}, \quad\left\langle\bar{Q}_{r}^{\alpha}\right\rangle=v \delta_{r}^{\alpha} .
$$

Although $v$ is undetermined so far because of the presence of a flat direction, it is not difficult to fix it to the GUT scale [10, [16]. In this vacuum, $\mathrm{SU}(3)_{C}$ is an unbroken linear combination of an $\mathrm{SU}(3)$ subgroup of the $\mathrm{SU}(5)_{\mathrm{GUT}}$ and the hypercolor $\mathrm{SU}(3)_{H}$, and $\mathrm{U}(1)_{Y}$ is that of a $\mathrm{U}(1)$ subgroup of the $\mathrm{SU}(5)_{\mathrm{GUT}}$ and the hyper $\mathrm{U}(1)_{H}$. The unification of the three gauge coupling constants of $\mathrm{SU}(3)_{C}, \mathrm{SU}(2)_{L}$ and $\mathrm{U}(1)_{Y}$ is practically achieved when the $\mathrm{SU}(3)_{H}$ and $\mathrm{U}(1)_{H}$ gauge interactions are enough strong at the GUT scale. The colored Higgs multiplets acquire a mass of the order of the GUT scale together with $Q_{\alpha}^{6}$ and $\bar{Q}_{6}^{\alpha}$. On the other hand, the Higgs doublets do not have such a large mass because they have no partners to form $Z_{4 R}$ invariant mass terms.

One can easily check that the $Z_{4 R}$ symmetry is free from the mixed anomalies with $\mathrm{SU}(5)_{\mathrm{GUT}}$ and $\mathrm{SU}(3)_{H}$ in the presence of the extra matter multiplets. It is also straightforward to check that the mixed gravitational anomaly and the cubic anomaly of the $Z_{4 R}$ symmetry are cancelled by adding some singlets.

Finally, we comment on the extra matter multiplets. For simplicity, we take one pair of $\Psi(5)$ and $\bar{\Psi}\left(\mathbf{5}^{*}\right)$ as the extra matter multiplets. The sum of their charges is restricted by Eq.(45): $x+\bar{x}=0$, while each charge remains undetermined from anomaly cancellation conditions. However, only the case that $x=-1$ and $\bar{x}=1$ turns out to be phenomenologically viable from the following arguments.

There can be four choices for the $Z_{4 R}$ charge $x$ of the extra matter multiplet $\Psi$, that is, $x=0,1,2,3$. In the case $x=\bar{x}=0$, the extra matter multiplets have the same quantum numbers as the Higgs, allowing such a interaction as $T T \Psi$ and $T \bar{F} \bar{\Psi}$. Then the $\mathrm{SU}(3)_{C}$ triplets of the extra matter multiplets behave like the colored Higgs, and lead to too fast proton decays. In the case $x=1$ and $\bar{x}=-1$, one of the three matter multiplet $\bar{F}$ forms a Planck mass term with the extra matter multiplets $\Psi$, while the

\footnotetext{
${ }^{9}$ There can be another choice that the extra matter multiplets have fractional charges. In this case the lightest extra matter particle is stable. However, its relic abundance is not negligible, so that this choice is excluded by the dark matter searches.
} 
remaining $\bar{\Psi}$ cannot form a Yukawa interaction $T \bar{\Psi} \bar{H}$. Therefore one of the down-type quarks and the charged leptons are massless. In the case that $x=2$ and $\bar{x}=-2$, the extra matter multiplets and the Higgs doublets form Planck scale mass terms as $\Psi \bar{H}$ and $\bar{\Psi} H$, so that no Higgs doublets remain at the weak scale. At last, it is found that the allowed choice is only that $x=-1$ and $\bar{x}=1$.

Since the extra matter multiplets $\Psi$ and $\bar{\Psi}$ have the $Z_{4 R}$ charges $x=-1$ and $\bar{x}=1$ respectively, they have the following Yukawa couplings and the mass terms

$$
W=y T \bar{F} \bar{H}+z T \bar{\Psi} \bar{H}+\langle W\rangle \Psi \bar{F}+\langle W\rangle \Psi \bar{\Psi} .
$$

Although there are mixings between the extra matter $\Psi$ and the matter multiplets $T$, these mixings are suppressed by $z\langle\bar{H}\rangle /\langle W\rangle$, so that their contributions to the FCNC processes are negligible. On the other hand, the Yukawa coupling $z T \bar{\Psi} \bar{H}$ could disturb the degeneracy among the masses of squarks and sleptons in the matter multiplets $T$, whose effects on the FCNC processes could be observed by future experiments.

\section{Conclusions}

In this paper we search for nonanomalous discrete R-symmetries in the MSSM and the GUT from a phenomenological point of view. We find that the nonanomalous discrete R-symmetry should be $Z_{9 R}$ or $Z_{18 R}$ in the MSSM. In the case of the GUT, on the other hand, we find that there must be some extra matter multiplets in order to cancel the anomalies and that only the $Z_{4 R}$ and $Z_{20 R}$ symmetries are allowed. The simplest choice of the extra matter multiplets is to take a pair of $\mathbf{5}$ and $\mathbf{5}^{*}$ of the $\mathrm{SU}(5)_{\mathrm{GUT}}$. These extra matter multiplets have masses of the order of the weak scale, and the presence of them is testable in future hadron collider experiments. As an example including the $Z_{4 R}$ symmetry, we give a unification model based on a semi-simple gauge group $\mathrm{SU}(5)_{\mathrm{GUT}} \times \mathrm{U}(3)_{\mathrm{H}}$.

We conclude this paper with a comment on the next-to-minimal SUSY SM (NMSSM) [17] with the gauge-mediated SUSY breaking (GMSB) [18]. In the GMSB, the NMSSM is an attractive mechanism generating the $\mu$-term because the Giudice-Masiero mechanism

\footnotetext{
${ }^{10}$ Note that the third term $\langle W\rangle \Psi \bar{F}$ can be absorbed into the last term $\langle W\rangle \Psi \bar{\Psi}$ by an appropriate redefinition of the multiplets $\bar{F}$ and $\bar{\Psi}$.
} 
does not work well. The Higgs sector in the NMSSM is $W=S H \bar{H}+S^{3}$, where $S$ is a singlet field. If the $Z_{N R}$ charge of $S$ is $2 \alpha$, the NMSSM is able to have the $Z_{4 R}$ or $Z_{20 R}$ symmetry. In the NMSSM with the GMSB, as is known well, some extra fields are necessary to couple with the singlet $S$ in order to obtain a viable spectrum [18, 19, 20]. It is very interesting that we have already introduced such extra fields as the extra matter multiplets $\Psi$ and $\bar{\Psi}$ in order to cancel the anomalies of the $Z_{4 R}$ and $Z_{20 R}$ symmetries.

\section{Acknowledgements}

The authors would like to thank T. Watari for useful discussions. This work was partially supported by the Japan Society for the Promotion of Science (K.K. and N.M.) and "Priority Area: Supersymmetry and Unified Theory of Elementary Physics (\# 707)" (T.Y.).

\section{References}

[1] J. Polchinski, String Theory, (Cambridge University Press, UK, 1998).

[2] Y. Imamura, T. Watari and T. Yanagida, hep-ph/0103251.

[3] S. Dimopoulos and H. Georgi, Nucl. Phys. B193, 150 (1981).

[4] N. Sakai and T. Yanagida, Nucl. Phys. B197, 533 (1982); S. Weinberg, Phys. Rev. D26, 287 (1982).

[5] G. Guidice and A. Masiero, Phys. Lett. B206, 1480 (1988).

[6] M.B. Green and J.H. Schwartz, Phys. Lett. B149, 117 (1984).

[7] L.E. Ibáñez and G.G. Ross, Phys. Lett. B260, 291 (1991); L.E. Ibáñez and G.G. Ross, Nucl. Phys. B368, 3 (1992); L.E. Ibáñez, Nucl. Phys. B398, 301 (1993).

[8] T. Yanagida, Phys. Lett. B344, 211 (1995); T. Hotta, K.-I. Izawa and T. Yanagida, Phys. Rev. D53, 3913 (1996); Phys. Rev. D54, 6970 (1996).

[9] J. Hisano and T. Yanagida, Mod. Phys. Lett. A10, 3097 (1995).

[10] K.-I. Izawa and T. Yanagida, Prog. Theor. Phys. 97, 913 (1997). 
[11] T. Yanagida, in Proc, of the Workshop on the Unified Theory and Baryon Number in the Universe, ed. O. Sawada and A. Sugamoto, (KEK report 79-18, 1979); M. Gell-Mann, P. Ramond and R. Slansky, in Supergravity, ed P. van Nieuwenhuizen and D.Z. Freedman, (North Holland, Amsterdam, 1979)

[12] T. Banks and M. Dine, Phys. Rev. D45, 1424 (1992).

[13] S. Dawson, Nucl. Phys. 261, 297 (1985).

[14] J. Ellis, G.B. Gelmini, J.L. Lopez and D.V. Nanopoulos, Nucl. Phys. B373, 399 (1992).

[15] G.B. Gelmini, P. Gondolo and S. Sarkar, Nucl. Phys. B392, 111 (1993).

[16] K.-I. Izawa, K. Kurosawa, Y. Nomura and T. Yanagida, Phys. Rev. D60, 115016 (1999).

[17] J. Ellis, J.F. Gunion, H.E. Haber, L. Roszkowski and F. Zwirner, Phys. Rev. D39, 844 (1989), and references therein.

[18] M. Dine and A.E. Nelson, Phys. Rev. D48, 1277 (1993); M. Dine, A.E. Nelson and Y. Shirman, Phys. Rev. D51, 1362 (1995); M. Dine, A.E. Nelson, Y. Nir and Y. Shirman, Phys. Rev. D53, 2658 (1996).

[19] K. Agashe and M. Graesser, Nucl. Phys. B507, 3 (1997).

[20] A. de Gouvêa, A. Friedland and H. Murayama, Phys. Rev. D57, 5676 (1998). 